\title{
Genetic Control of Fertility in Streptomyces coelicolor A3(2): New Kinds of Donor Strains
}

\author{
By A. VIVIAN* AND D. A. HOPWOOD \\ John Innes Institute, Norwich NOR $70 F$
}

(Received I I December I972)

\begin{abstract}
SUMMARY
New types of donor strains have been obtained by indirect selection from an IF strain bearing the autonomous plasmid SCP I. The new donors differ from the previously known type of donor (NF) in being, usually, unstable, segregating numerous IF and UF variants, and in their pattern of donation to UF strains (recipient strains lacking SCP I). Whereas the chromosome fragments donated by NF strains have neither end at a constant position, those transferred by the new donors have one constant and one variable end. The new donors are designated according to the marker donated with the highest frequency - that is, nearest to the constant end of the chromosome fragments. It is postulated that they arise by interaction between SCP I and the chromosome, perhaps to produce substituted plasmids analogous to F prime strains of Escherichia coli, which may mobilize the chromosome by donor crossing-over. Most of the new donors had a white colony phenotype, although producing apparently normal spores.
\end{abstract}

\section{INTRODUCTION}

IF strains of Streptomyces coelicolor A3(2) harbour a plasmid SCP I (Vivian, I97I). Loss of SCP I results in the production of UF variants (Hopwood, Harold, Vivian \& Ferguson, 1969). NF strains act as donors of chromosome fragments to both IF and UF strains (Hopwood et al. 1969; Vivian \& Hopwood, 1970).

The presence of SCP I in IF strains causes them to excrete a diffusible substance which inhibits the production of aerial mycelium by UF strains (Vivian, 197I). Since NF strains also inhibit aerial mycelium production by UF and, like IF strains, are resistant to the inhibitor (A. Vivian \& D. A. Hopwood, unpublished results; see Hopwood, 1972) they presumably also harbour SCP I. However, since they do not readily lose it to produce UF variants (Vivian \& Hopwood, 1970), the plasmid is unlikely to be autonomous as it presumably is in IF strains; it is probably associated with the chromosome in the 9 o'clock position (Vivian, I97I).

Two striking features characterize the behaviour of NF donor strains when crossed with UF recipients (Hopwood et al. 1969): the crosses are 'ultra-fertile', in that no (or very few) progeny of either parental phenotype are found amongst the spores produced on the mixed culture; and there is a bidirectional gradient in the frequencies of different donor markers amongst the progeny. The frequency is inferred to be $100 \%$ for the 9 o'clock region of the NF genome, falling sharply in both clockwise and anticlockwise directions to values as low as $\mathrm{I} \%$ or less for distant markers such as $\arg A$ or $c y s D$. (See the linkage map in Fig. I.)

$\mathrm{NF}$ arose spontaneously once within the pedigree of recombinant strains derived from the IF wild-type A3(2) (Vivian \& Hopwood, 1970). It follows from what has been said above that this

* Present address: School of Biological Sciences, Thames Polytechnic, London, SEI 8 6PF. 
event is most likely to have involved the integration in some way of SCP I into the chromosome in the 9 o'clock position. Thus it should be possible experimentally to derive NF variants from an IF strain by selecting the products of integration. Indeed it might be possible to find such variants enriched amongst the progeny of an IF $\times$ UF cross on the assumption that a part, at least, of the fertility of such a cross would derive from NF variants originating spontaneously within the IF culture. However, attempts to find such NF variants failed (A. Vivian, unpublished results). We therefore tried to isolate NF, or other possible donor variants, from an IF culture by an indirect, rather than a direct, selection procedure. In the course of this work, a group of new donor types was discovered, some of which are described in the present paper. NF variants were not isolated in one step from IF strains, but strains possibly identical with NF were derived by further evolution from the primary donors.

\section{METHODS}

General. Complete (CM) and minimal (MM) media and standard cultural techniques were those described by Hopwood (1967).

Strains. The strains, which are all mutational and recombinational derivatives of Streptomyces coelicolor A3(2) (Hopwood, I959), are listed in Table I and the locations of markers on the linkage map are in Fig. I.

Ultraviolet irradiation (u.v.). This was done by the method of Harold \& Hopwood (1970). The dose was about $3800 \mathrm{ergs} / \mathrm{mm}^{2}$.

Testing of fertility by 'plate crossing'. This was done by a modification of the indirect selection procedure of Sermonti \& Casciano (1963) as described by Hopwood et al. (I969) and Vivian \& Hopwood (1970).

Procedure for the isolation of donor strains. The practical details of the method were essentially the same as in the isolation of UF variants from an IF strain (Hopwood et al. 1969) except that the tester strain was UF instead of NF. For reasons outlined below, the UF tester strain carried the marker $p a b A I$, located just clockwise of the 9 o'clock region.

Suitable dilutions of a spore suspension of strain I 2 pheAI IF, either u.v. treated or untreated, were plated to give about 200 colonies/dish. After about 5 days of incubation, when the colonies were sporulating vigorously, they were replicated to plates of CM on which a dense spore suspension of the UF tester strain A574 pabAr had been spread. The resulting 'plate crosses', after incubation for 4 days, were replica plated to $M \mathrm{M}$ to select recombinants inheriting $p a b A^{+}$from the IF parent and $p h e A^{+}$from the UF.

The yield of recombinants from IF $\times$ UF crosses does not usually exceed $\mathrm{IO}^{-4}$, so that most of the tested colonies gave few or no selected recombinants. However, sectors within certain colonies on the original plates were shown by the test to donate $p a b A^{+}$with a high frequency, and were isolated as presumptive donors.

\section{RESULTS}

Isolation and preliminary characterization of the new donor strains

The NF/IF difference has been located chromosomally at 9 o'clock on the linkage map (Vivian \& Hopwood, I970). NF donates markers to UF with frequencies which decline with increasing distance of the marker in both directions from the 9 o'clock region. The difference in frequency of inheritance of two markers, such as nic $A$ and $c y s D$, may be more than Ioofold (Hopwood et al. 1969). Consequently it might be expected that the donor marker employed in the selection procedure would influence the ability to detect NF strains within 
IF populations. A counterselectable UF marker as close as possible to the 9 o'clock region was therefore chosen, on the assumption that the wild-type allele of such a marker would be more frequently transferred by a donor strain resembling the type NF.

All the strains described here were isolated from strain 12 after u.v. treatment, on the basis of $p a b A^{+}$donation to UF strain A574. After u.v. irradiation to a survival of $1 \cdot 6 \times 10^{-3}$, I333 I colonies yielded 23 donor strains $(0.17 \%)$, whereas without irradiation no donors were recognized amongst 3722 colonies. Thus u.v. probably increased the frequency of origin of donor strains. In contrast to experience in the isolation of UF variants from IF (Hopwood et al. 1969), entire colonies donating with a high frequency were not found; always the donors formed sectors of variable extent within an otherwise poorly donating colony, a finding probably related to the instability of most of the donor strains (see below). The sectors were easily distinguished because they were white, in contrast to the normal grey colour of the IF parent culture.

\section{Instability of most of the donor strains}

Donor cultures isolated after repeated single colony streaking were, with one exception (A608), unstable, yielding grey, usually non-donor colonies. Three cultures were investigated in some detail.

A608 was apparently very stable (Table 2) yielding no grey or non-donor colonies out of more than 6000 colonies tested. A dose of u.v. which would have induced around $1 \%$ UF segregants from the corresponding IF strain (I2) caused no instability of A608. A608, however, did give rise with a low frequency (I out of 2863 colonies in one experiment) to at least one new kind of strain which, unlike A608 (see below), donated $u r a A^{+}$with high frequency.

A607 was very unstable (Table 2), yielding grey segregants with a high spontaneous frequency which was clearly increased by u.v. irradiation. The grey segregants were of three kinds: UF, IF and donors resembling $\mathrm{A} 607$ but being grey and having a slightly reduced growth rate. The relative proportions of the three kinds of grey segregants in one experiment were approximately $60 \%$ UF, $20 \%$ IF and $20 \%$ grey donors. Fig. 2 shows colonies of $\mathrm{A} 607$ spontaneously giving rise to grey sectors. The grey donor segregants were in turn unstable, producing white segregants, easily visible as faster-growing sectors on the colonies (Fig. 3); they did not give rise to UF or IF colonies with measurable frequency. Grey donors also gave rise, at a low frequency (about $0.4 \%$ ), to $\mathrm{uraA}^{+}$donors. At least some of the white segregants from the grey donors repeated the behaviour of the original white $A 607$ strain.

A6 10 resembled $A 607$ in being highly unstable (Table 2), yielding grey segregants, the great majority of which were UF strains; however, grey IF segregants were also present. In one experiment, $97 \%$ of the grey segregants were UF and $3 \%$ were IF.

The nature of the secondary donor types derived from the primary donors will be considered after the patterns of donation of the latter have been described.

\section{Different patterns of donor ability towards UF strains}

Two main types of donor strain were distinguished on the basis of their pattern of marker donation. Of the 23 strains, all except one donated, with detectable frequency, $p a b A^{+}$and markers clockwise from it in the top half of the map. As an example, Fig. 4 shows a visual comparison of the relative donor capabilities of NF strains with those of one of the new donor strains, A620. It can be seen that NF strains donated all the markers tested with a relatively high frequency. (In the plate-cross procedure, the test is 'saturated' so that donation by $\mathrm{NF}$ of a marker even far from the 9 o'clock region is sufficient to give a nearly confluent patch of recombinants.) A620 donated $p a b A^{+}$with a frequency indistinguishable from NF, but 
Table I. Characteristics of strains

Relevant phenotype: markers in order clockwise from 9 o'clock

\begin{tabular}{|c|c|c|c|c|c|c|c|c|c|c|c|c|}
\hline Strain & $p a b A I$ & ade-vIo & cys $A I 5$ & proAI & his $A \mathrm{I}$ & $\arg A I$ & $c y s D I 8$ & $m t h B 2$ & $\operatorname{str} A I$ & pheAr & uraAI & Fertility $\dagger$ \\
\hline $\mathrm{A} 3(2)^{*}$ & + & + & + & + & + & + & + & + & S & + & + & IF \\
\hline 12 & + & + & + & + & + & + & + & + & $\mathrm{S}$ & - & + & IF \\
\hline 1098 & + & + & + & + & + & + & + & + & S & - & + & UF \\
\hline II 4 I & + & + & + & + & + & + & + & + & $\mathbf{S}$ & - & + & $\mathrm{NF}$ \\
\hline II 90 & + & + & + & + & - & + & + & + & $\mathbf{R}$ & + & - & UF \\
\hline A332 & + & + & + & + & + & + & + & + & $\mathbf{S}$ & - & + & $\mathrm{NF}$ \\
\hline A372 & + & + & - & - & + & - & + & + & $\mathbf{R}$ & + & - & UF \\
\hline A450 & + & + & + & + & - & + & + & + & $\mathbf{S}$ & + & + & UF \\
\hline A574 & - & + & + & + & + & + & + & + & $\mathbf{S}$ & + & + & UF \\
\hline A585 & + & - & + & + & + & + & - & - & $\mathbf{S}$ & + & + & UF \\
\hline A607 & + & + & + & + & + & + & + & + & $\mathbf{S}$ & - & + & $p a b A^{+}$donor \\
\hline A608 & + & + & + & + & + & + & + & + & $\mathbf{S}$ & - & + & $p a b A^{+}$donor \\
\hline A6IO & + & + & + & + & + & + & + & + & $\mathbf{S}$ & - & + & $u_{r a A^{+}}$donor \\
\hline A6I 7 & - & + & + & + & + & - & + & + & $\mathbf{R}$ & + & - & UF \\
\hline A620 & + & + & + & + & + & + & + & + & $\mathbf{S}$ & - & + & $p a b A^{+}$donor \\
\hline A634 & + & + & - & - & + & - & + & + & $\mathbf{R}$ & + & + & Presumptive NF \\
\hline
\end{tabular}

Table 2. Stability of the new donor strains

The tester strain for fertility tests was 1190.

$\begin{array}{ccccc}\text { Strain } & \text { Treatment } & \text { Survival }(\%) & \text { Total colonies } & \begin{array}{c}\text { Grey segregants } \\ (\%)\end{array} \\ \text { A607 } & \text { None } & 100 & 4085 & 48 \\ & \text { u.v. } & \mathrm{I} \cdot \mathrm{I} & 7113 & 96 \\ & \text { None } & 100 & 1133 & 63 \\ & \text { u.v. } & \mathrm{I} \cdot 7 & 195 \mathrm{I} & 9 \mathrm{I} \\ & \text { None } & 100 & 415 & \mathrm{I} \\ & \text { u.v. } & \mathrm{I} \cdot 4 & \mathrm{I} 254 & 17 \\ \mathrm{~A} 608 & \text { None } & 100 & 1066 & 0 \\ & \text { u.v. } & 2 \cdot 6 & 2863 & 0 \\ & \text { None } & 100 & 1914 & 0 \\ & \text { u.v. } & 2 \cdot 0 & 452 & 0 \\ \text { A610 } & \text { None } & 100 & 1875 & 5 \\ & \text { u.v. } & 0 \cdot \mathrm{I} & 237 & 23 \\ & \text { None } & 100 & 1757 & 20 \\ & \text { u.v. } & 0 \cdot 2 & 336 & 4 \mathrm{I} \\ & \text { None } & 100 & 857 & 90 \\ & \text { u.v. } & 0 \cdot 5 & 542 & 98\end{array}$

showed successively lower donation frequencies for $a d e-v I O^{+}$and $h i s A^{+}$. The plate test showed no detectable level of donation by $\mathrm{A} 620$ of $u \mathrm{raA} A^{+}$or $c y s D^{+}$, which span the group of markers in the lower half of the linkage map. Clearly A620 is quite distinct from NF, which donates all the markers between $u r a A$ and $c y s D$ with an appreciable frequency.

A series of crosses between the donor strains and UF strain A372 was made and the resulting spores were plated on various media, selecting $p h e A^{+}$from the UF strain with variation of the donor marker selected. Table 3 shows the numbers of selected recombinants obtained 


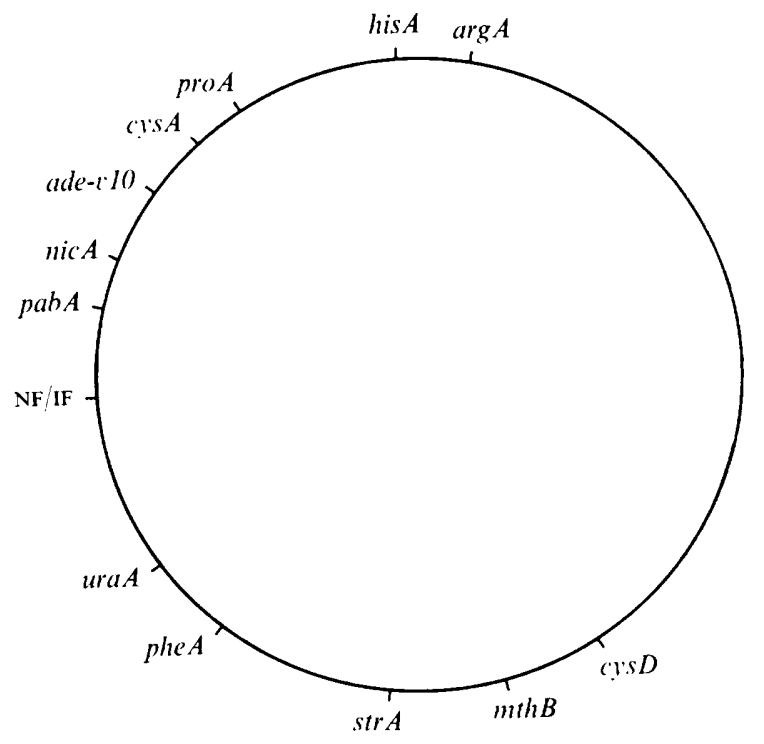

Fig. I. Location of markers. Locus symbols have conventional meaning (Hopwood, I967). NF/IF denotes the presumed attachment site of the plasmid SCP I at 9 o'clock on the linkage map in NF strains.

for $c y s A^{+}$compared with those for $\mathrm{uraA}^{+}$. As shown by the fourth column in Table 3, different donor strains varied widely in the ratio of $c y s A^{+}: u r a A^{+}$donation. Strains A607, A608 and A620 all donated $c y s A^{+}$with much greater frequency than $u r a A^{+}$, although varying considerably amongst themselves, while strain A6Io donated $u \mathrm{raA} A^{+}$much more frequently than $c y s A^{+}$. The first group of strains will be called ' $p a b A^{+}$donors', while A6ro is a ' $u r a A^{+}$ donor'. Variation in the ratio of $c y s A^{+}: u r a A^{+}$donation between different $p a b A^{+}$donors may reflect different frequencies of origin, within the donor cultures, of secondary donors transferring $\mathrm{uraA}^{+}$with high frequency (see below).

\section{Genetic analysis of the pab $A^{+}$donor $\mathrm{A} 607$}

Table 4 shows the pooled results of crosses of $\mathrm{A} 607$ and of a grey donor segregant of $\mathrm{A} 607$ with A372 UF; selection was for phe $A^{+}$from the UF and either $c y s A^{+}$or $\arg A^{+}$from the donor. The two crosses gave homogeneous results. The effect of the different selections is seen in the allele ratios amongst the progeny, recorded in the diagrams in Table 4 . With selection for cys $A^{+}$, only $14 \%$ of the progeny also inherited $\arg A^{+}$, but $74 \%$ of $\arg A^{+}$progeny were cys $A^{+}$. This result indicates a unidirectional gradient of donor allele frequencies decreasing clockwise from cys $A$ (and presumably from $p a b A$ ), which may be thought of as proximal markers, towards more distal markers, such as $\arg A$.

The data in the right-hand side of Table 4 , in which the donor marker $\arg A^{+}$is selected, indicate that this gradient is not due solely to a reduced probability of transfer to the zygotes of $\arg A^{+}$as compared with $c y s A^{+}$, although this is a contributory factor. In addition there must be an enhanced probability of inheritance by recombinants of donor proximal markers included in the zygotes. This is indicated by a consideration of the patterns of crossing-over needed to explain the different segregant phenotypes. Since the donor allele $\mathrm{uraA}^{+}$is not found in the progeny we can assume that the donor chromosome fragments in the zygotes do not include the uraA locus, but end somewhere in the interval cys $A-u r a A$. Thus the region 


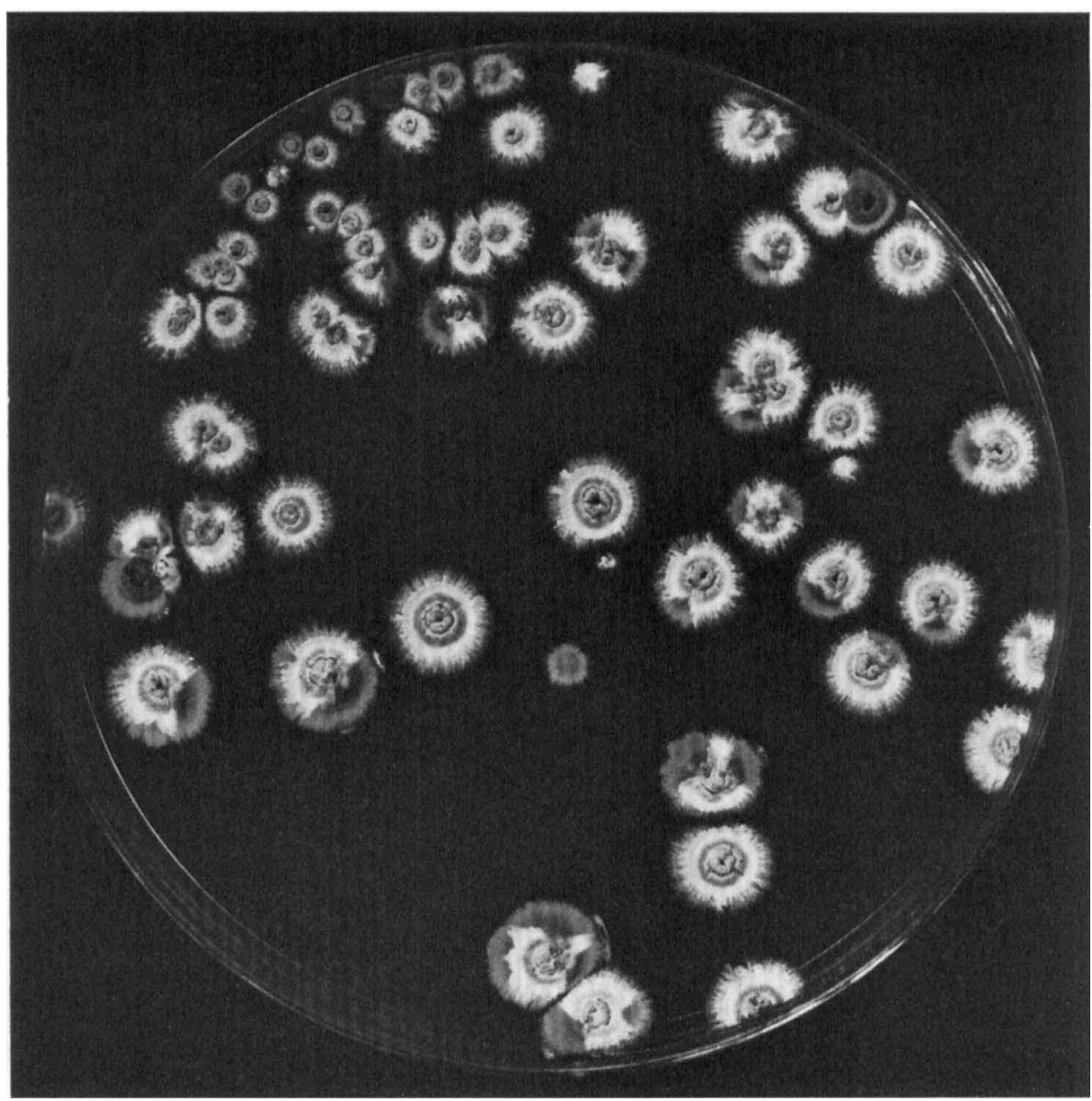

Fig. 2. Colonies of the $p a b A^{+}$donor $\mathrm{A} 607$ grown for 7 days on supplemented minimal medium. Note the white phenotype of most of the colonies, many of which are seen to be giving rise to grey sectors, of growth rate similar to that of the original strain. These sectors, when picked off and fertility tested, are found to consist mainly of UF segregants, with some IF. Grey donor segregants (Fig. 3) do not appear as sectors because of their reduced growth rate.

of the recipient (UF) chromosome starting from a point anticlockwise of the end of the donor fragments must be inherited; with this particular selection, the donor allele $\arg A^{+}$would also be obligate. If we consider the three map intervals between $\arg A$ and $u r a A$ - that is, intervals 2, 3 and $(4+5)$ - three of the four possible segregant phenotypes would represent single crossovers in each of these three map intervals while the fourth would represent the triple crossover class. In fact the presumptive triple crossover class, prourastr, has a frequency (18) even higher than that of one of the presumptive single crossover classes, cys urastr (frequency I6).

This apparent contradiction can be resolved by postulating that a point near the proximal end of the donor chromosome fragment is obligatorily inherited by the progeny, just as the 9 o'clock region of the donor NF chromosome is inherited by all progeny in NF $\times$ UF crosses (Hopwood et al. 1969: see Introduction). On this interpretation, crossing-over in a minimum of two regions is required to produce any viable progeny: clockwise of $\arg A^{+}$(interval $\mathrm{r}$ ), 


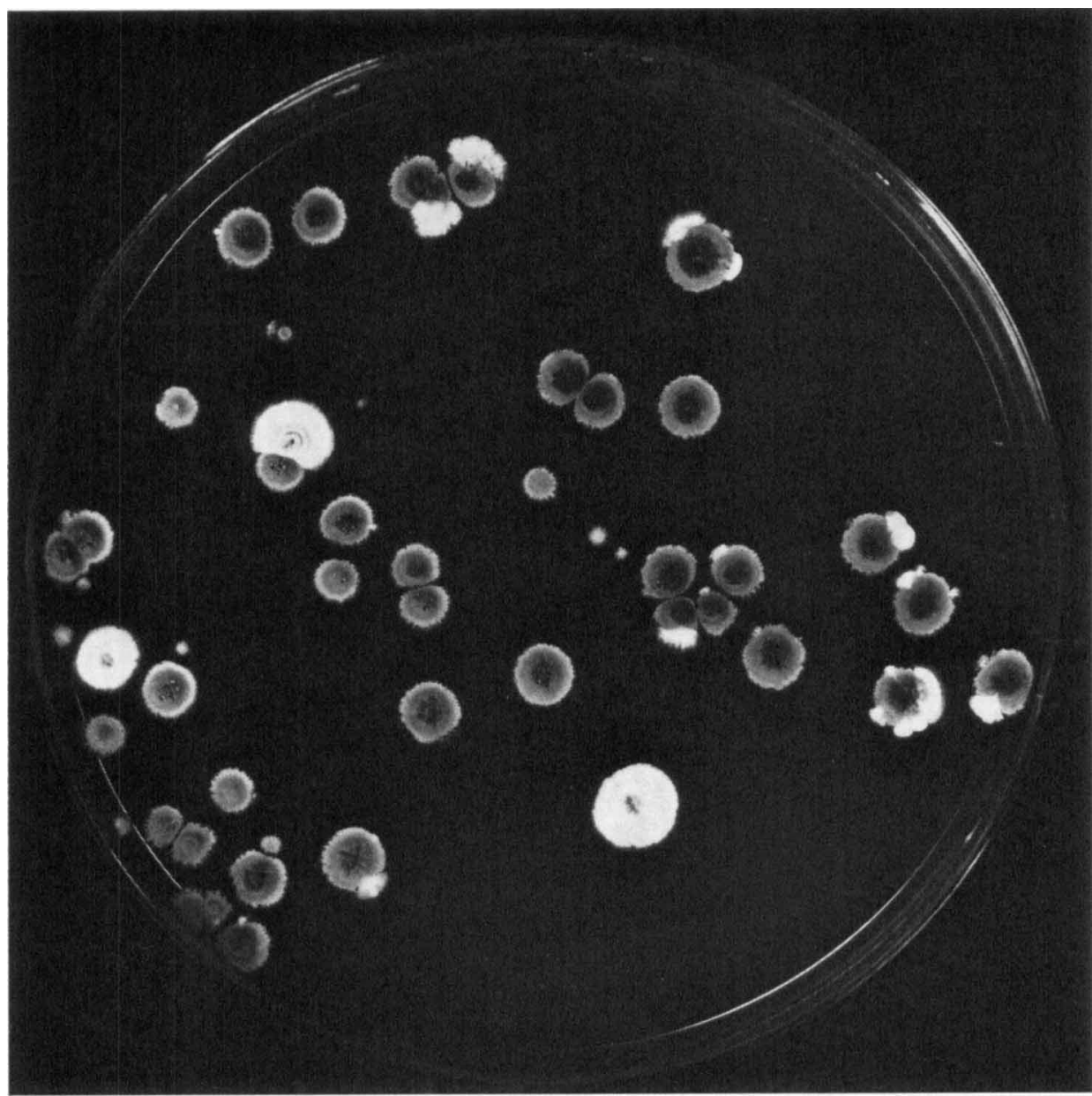

Fig. 3. Colonies of the grey $p a b A^{+}$donor derived from $A 607$ grown under identical conditions to those of Fig. 2. Note the slower growth rate of the grey colonies and their production of faster growing white sectors. These, on fertility testing, are found to be donors resembling A607.

and anticlockwise of the obligatorily inherited point just defined (interval 5). One of the four possible segregant phenotypes (urastr) is thus a non-crossover class in respect of the other three intervals, and it has a high frequency, while the other three classes, with much lower frequencies, represent the three possible double crossover classes in respect of these intervals (see the right-hand part of Table 4). The data obtained by selecting the donor marker $c y s \mathrm{~A}^{+}$ (left-hand side of Table 4) are also consistent with this interpretation although they cannot provide any evidence for the obligate inheritance of a proximal part of the donor fragment since the selected $c y s A^{+}$is the most proximal marker included on the fragment.

A further feature of crosses involving the new donor A607 is seen in a cross analysed nonselectively (Hopwood et al. 1969). The cross was ultra-fertile since only I colony out of 97 was parental both for markers and fertility type (Table 5). The only standard marker donated with a high enough frequency to be detected amongst the sample of progeny was $p a b A^{+}$, inherited by $33 \%$ of the progeny. This confirms the conclusion from selective analysis that A607 does not donate $u \mathrm{raA}^{+}$with a high frequency and that there is therefore a unidirectional 

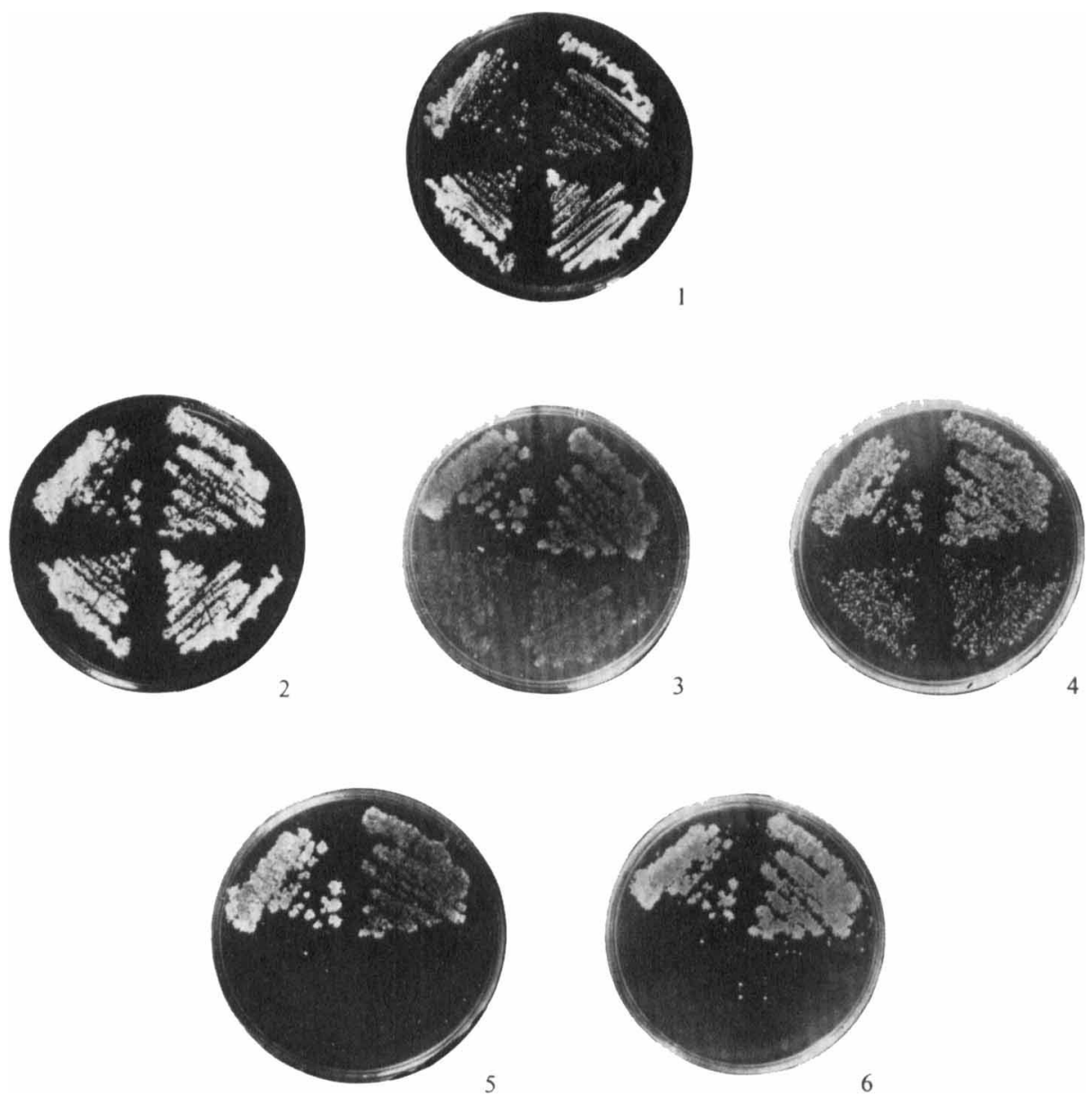

Fig. 4. Comparison of donor properties of a $p a b A^{+}$donor and NF strains. Plate I, of non-selective medium, bears strains as follows: top left, A332 NF; top right, I I $41 \mathrm{NF}$; bottom left and right, two separate single colonies of $\mathrm{A} 620 \mathrm{pab} \mathrm{A}^{+}$donor. This plate was replicated separately to three plates of $C M$ each spread with a dense spore suspension of a different UF tester strain. The resulting 'plate crosses' (not shown) were then replicated to appropriate test media, all selecting for $p h e A I^{+}$from the UF and each selecting a different marker from the donor, as follows. Plate $2 ; \mathrm{pabAI}^{+}$(UF tester A574); plate 3, ade-vIO (A585); plate 4, his $\mathrm{AI}^{+}$(I I90); plate 5, cysDI8+ (A585); plate 6, uraAI $\mathrm{II}^{+}$ (I 190). It can be seen that, whereas the two NF strains donate all the markers efficiently, the frequency of donation of different markers by A620 clearly falls in a clockwise direction ( $p a b \mathrm{AI}^{+}, a_{d e-}$ $\mathrm{vIO}^{+}$, his $\mathrm{AI}^{+}$; plates 2, 3, 4 respectively); cys $\mathrm{Dr} 8^{+}$and $\mathrm{uraAI}^{+}$(plates 5 and 6) are donated with a frequency too low to be detected by this test.

gradient of donation of markers in the top half of the map falling off sharply in a clockwise direction.

\section{Genetic analysis of the uraA ${ }^{+}$donor A610}

Table 6 shows the results of a cross A6IO $\times$ A372. Both selections, for donor markers $c y s A^{+}$ or $\arg A^{+}$, show high frequencies of $u r a A^{+}$inheritance amongst the progeny, in sharp contrast to the results obtained from the corresponding cross of A607 (Table 4). However, the relative frequencies of $c y s A^{+}$and $\arg A^{+}$in the two selections are very similar to those given by $\mathrm{A} 607$. 
Table 3. Comparison of donor abilities of different donor strains crossed with UF strain $\mathrm{A} 372$

Each line represents data from a single cross. The selected allele from strain A372 was always phe $\mathrm{AI}^{+}$

Colony counts/unit volume

of spore suspension

Selected donor allele

Donor strain

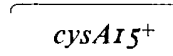

ura $A I^{+}$

A607

A607

56500

A608

56000

A620

9700

2600

A610

305

73
190
1650
55
4235

$\overbrace{\text { cys AI } 5^{+} / \text {uraAI }^{+}}^{\text {Ratio }}$
$\begin{gathered}779 \\ 296 \\ 5.9 \\ 47 \\ 0.07\end{gathered}$

Table 4. Effect of varying the imposed selection on the segregation of non-selected alleles in a cross of $\mathrm{A} 607$ ( $p a b A^{+}$donor: outer circle) with $\mathrm{A} 372$ (UF: inner circle)

Selected alleles are indicated by triangles.

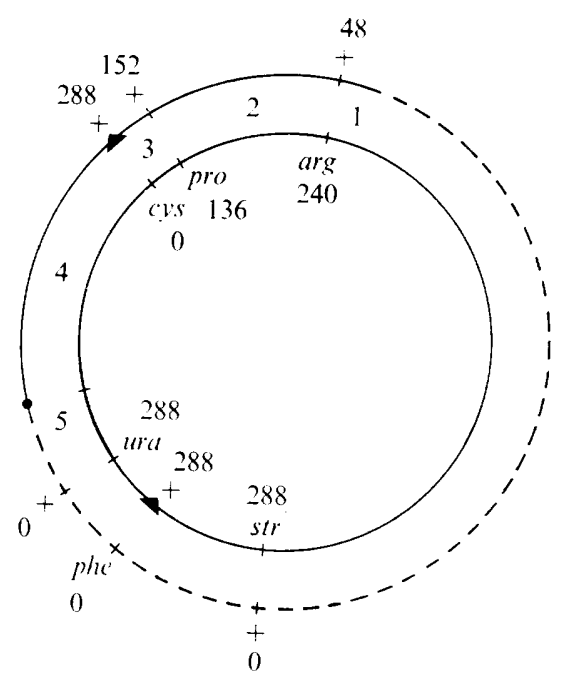

Selected donor allele $\operatorname{cys} \mathrm{A}^{+}$

$\begin{array}{clr}\begin{array}{c}\text { Crossovers } \\ \text { in intervals }\end{array} & \text { Phenotype* }^{*} & \begin{array}{c}\text { Observed } \\ \text { number }\end{array} \\ \mathrm{I}, 5 & \text { ura str } & 43 \\ 2,5 & \text { arg ura str } & 109 \\ 3,5 & \text { pro arg ura str } & 13 \mathrm{I} \\ \mathrm{I}, 2,3,5 & \text { pro ura str } & 5 \\ & \text { Total } & 288\end{array}$

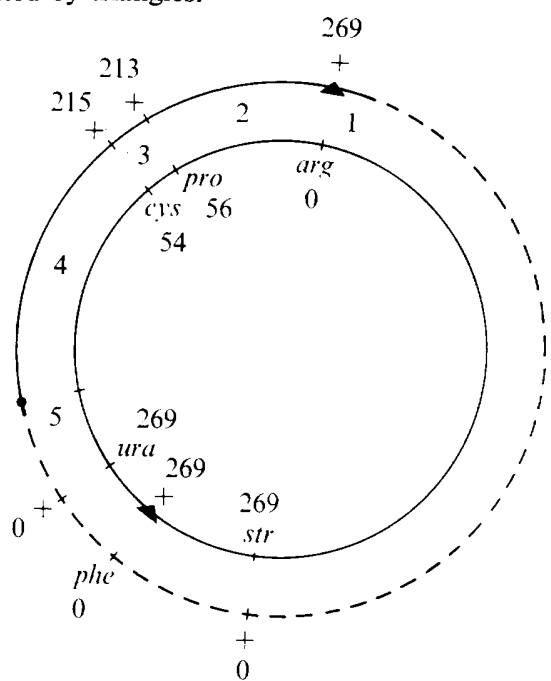

Selected donor allele $\arg \boldsymbol{A}^{+}$

$\begin{array}{clr}\begin{array}{c}\text { Crossovers } \\ \text { in intervals }\end{array} & \text { Phenotype* } & \begin{array}{r}\text { Observe } \\ \text { number }\end{array} \\ \mathrm{I}, 5 & \text { ura str } & \mathrm{I} 97 \\ \mathrm{I}, 2,3,5 & \text { pro ura str } & 18 \\ \mathrm{I}, 2,4,5 & \text { cys pro ura str } & 38 \\ \mathrm{I}, 3,4,5 & \text { cys ura str } & \mathrm{I} 6 \\ & & \end{array}$

* In this and subsequent tables, wild-type alleles are omitted in phenotypic designations. 
Table 5. Non-selective analysis of a cross of $\mathrm{A} 607$ (pabA $A^{+}$donor: outer circle) with A6I 7 (UF: inner circle)

Numbers represent the allele frequencies amongst the non-parental progeny. The point indicated on the donor genome between $\mathrm{pab}^{+}$and $u \mathrm{ra}^{+}$represents the region deduced to be obligatorily inherited by non-parental progeny (see text).

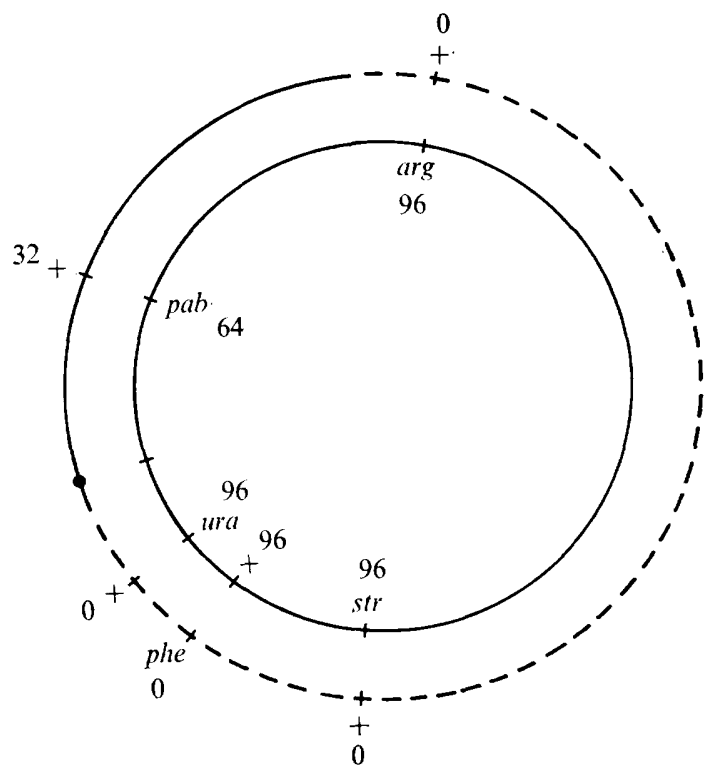

\begin{tabular}{|c|c|}
\hline $\begin{array}{l}\text { Segregant phenotype } \\
\text { phe }\end{array}$ & $\begin{array}{l}\text { Observed number } \\
\text { I Parental }\end{array}$ \\
\hline $\begin{array}{l}\text { arg ura str } \\
\text { arg pab ura str* }\end{array}$ & $\left.\begin{array}{l}32 \\
64\end{array}\right\}$ Non-parental \\
\hline Total & 97 \\
\hline
\end{tabular}

* These segregants had donor fertility (tester strains A450, I098 and II4I).

The data in Table 6 may be considered in terms of the crossovers needed to produce each class of segregant phenotype, as was done for the A607 cross (Table 4). Again, evidence for an obligate inheritance of a proximal region of the donor fragment is found, with the end in this case lying between the loci $u r a A$ and $p h e A$. The evidence for such obligate inheritance in this cross is even more direct since the frequency of the non-selected proximal donor allele $\mathrm{uraA}^{+}$is more than ten times higher than that of the recipient allele $u \mathrm{raA}$, although it is separated from the only donor marker for which conscious selection was made, $\arg A^{+}$, by nearly half the linkage map.

The results of non-selective analysis of a cross A6IO $\times$ A6I 7 are in Table 7 . The cross, like that involving $\mathrm{A} 607$ (Table 5), was ultra-fertile. However, in this case $u \mathrm{raA}^{+}$was donated to more than $50 \%$ of the progeny and $p a b A^{+}$to $19 \%$. The unidirectional gradient of donor marker frequencies is seen very clearly in this cross, since donor markers anticlockwise of $\mathrm{uraA}^{+}$, even at a locus as close as phe $A$, have undetectably low frequencies. 
Table 6. Effect of varying the imposed selection on the segregation of non-selected alleles in a cross of $\mathrm{A} 6 \mathrm{IO}$ (uraA ${ }^{+}$donor: outer circle) with $\mathrm{A} 372$ (UF: inner circle)

Selected alleles are indicated by triangles.

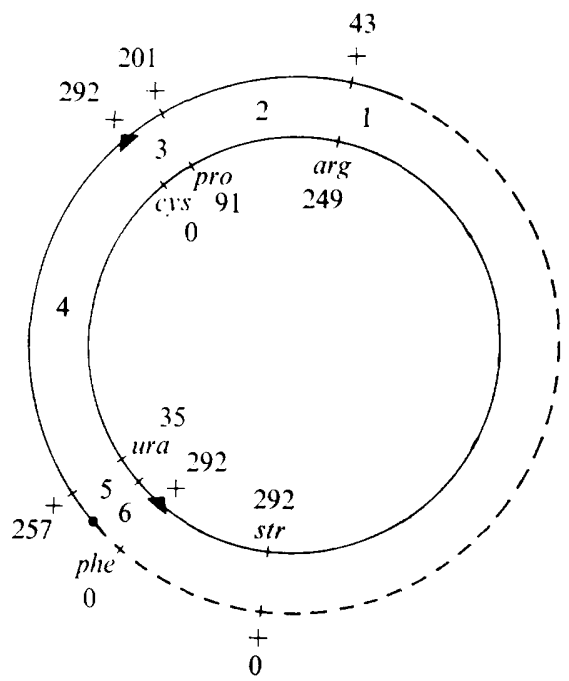

Selected donor allele cys $A^{+}$

$\begin{array}{llr}\begin{array}{l}\text { Crossovers } \\ \text { in intervals }\end{array} & \text { Phenotype } & \begin{array}{r}\text { Observed } \\ \text { number }\end{array} \\ \mathrm{I}, 6 & \text { str } & 4 \mathrm{I} \\ 2,6 & \text { arg str } & \mathrm{I} 39 \\ 3,6 & \text { pro arg str } & 77 \\ \mathrm{I}, 4,5,6 & \text { ura str } & 2 \\ 2,4,5,6 & \text { arg ura str } & \mathrm{I} 9 \\ 3,4,5,6 & \text { pro arg ura str } & \mathrm{I} 4 \\ \mathrm{I}, 2,3,6 & \text { pro str } & 0 \\ \mathrm{I}, 2,3,4,5,6 & \text { pro ura str } & 0 \\ & \text { Total } & 292\end{array}$

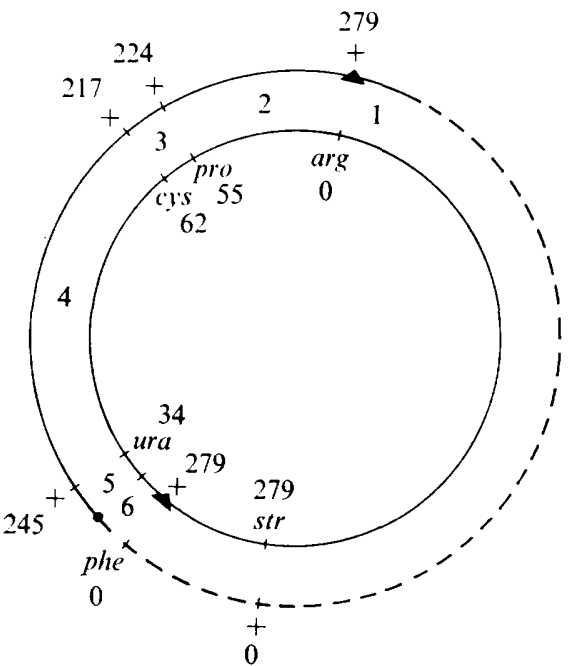

Selected donor allele $\arg A^{+}$

\begin{tabular}{llr}
$\begin{array}{l}\text { Crossovers } \\
\text { in intervals }\end{array}$ & \multicolumn{1}{c}{ Phenotype } & $\begin{array}{c}\text { Observed } \\
\text { numbers }\end{array}$ \\
I, 6 & str & 190 \\
I, 2, 3, 6 & pro str & 8 \\
1, 2, 4, 6 & cys pro str & 33 \\
I, 2, 5, 6 & cys pro ura str & 14 \\
I, 3, 4, 6 & cys str & 14 \\
I, 3, 5, 6 & cys ura str & $\mathrm{I}$ \\
I, 4, 5, 6 & ura str & 19 \\
I, 2, 3, 4, 5,6 & pro ura str & 0 \\
& Total & 279
\end{tabular}

\section{Properties of the recombinants from new donor $\times$ UF crosses}

Samples of progeny from the crosses shown in Tables 4 and 6, involving $p a b A^{+}$donor A607 and $u \mathrm{raA}^{+}$donor A6IO respectively, were characterized in respect of their fertility type. The great majority of the selected recombinants were unstable donors apparently resembling their donor parent except in having a new genotype. They readily lost donorability, and in doing so their genotype in respect of the standard markers in the cross remained constant: they did not lose expression of the selected marker (cys $A^{+}$or $\left.\arg A^{+}\right)$derived from their donor parent. Thus they behaved as normal haploid recombinants. Many, usually the majority, of the non-recombinant progeny bearing the markers of the UF parent had also become unstable donors: only a few were still UF. This finding is compatible with the results of the non-selective crosses in Tables 5 and 7. The interpretation is that the donor progeny with the genotype of the UF parent differ from the overtly recombinant progeny only in the particular map intervals in which crossing-over occurred to produce them. 
Table 7. Non-selective analysis of a cross of $\mathrm{A} 6 \mathrm{IO}$ (uraA $A^{+}$donor: outer circle) with A6I 7 (UF: inner circle)

Numbers represent the allele frequencies amongst the non-parental progeny. The point indicated on the donor genome between $u \mathrm{ra}^{+}$and phe represents the region deduced to be obligatorily inherited by non-parental progeny (see text).

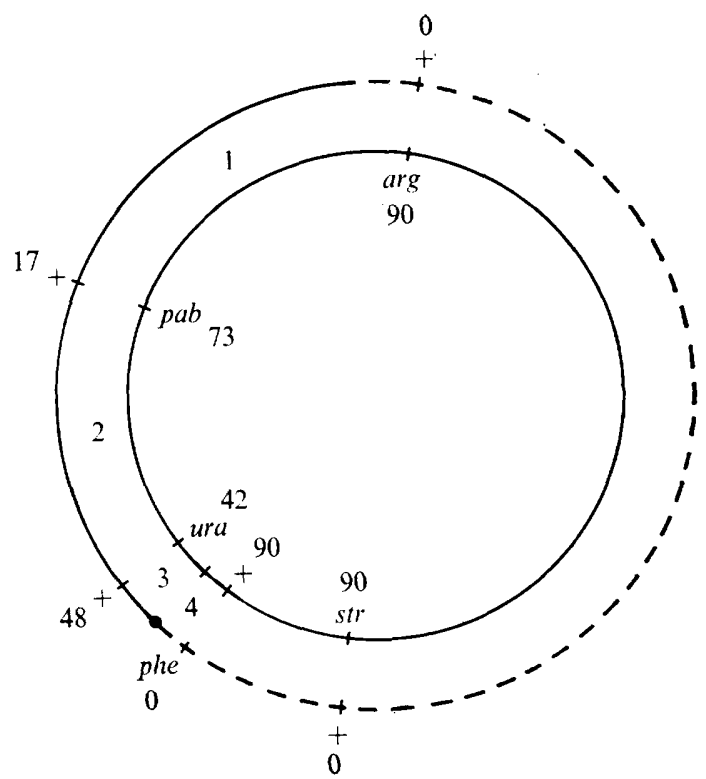

$\begin{array}{cl}\text { Crossovers in intervals } & \text { Segregant phenotype } \\ & \text { phe } \\ \mathrm{I}, 4 & \text { arg str } \\ 2,4 & \text { arg pab str } \\ 3,4 & \text { arg pab ura str } \\ \mathrm{I}, 2,3,4 & \text { arg ura str }\end{array}$

Total

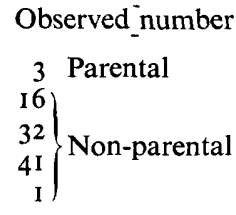

93

Selection of strains resembling $\mathrm{NF}$ from the new donors

Donor strains having most or all of the properties of NF strains have been derived from the new donors in two ways. The first involved selection. As we have seen, $p a b A^{+}$donors like $\mathrm{A} 607, \mathrm{~A} 608$ and $\mathrm{A} 620$ donate $u \mathrm{raA}^{+}$with a frequency barely detectable by the plate-crossing procedure (Fig. 4). However, when colonies of such strains were plate-crossed with strain I I90 (hisAI uraAI strAI UF) and selection was made alternatively for uraA $A^{+}$or $h i s A^{+}$ from the donor (and phe $A^{+}$from the recipient), a small minority of colonies was detected that donated $u r a A^{+}$with a frequency apparently as high as that of $h i s A^{+}$donation by the majority of the population, and they continued to donate his $A^{+}$with high frequency. The results of non-selective analysis of two of these donors with A6I 7 clearly showed that the donors resembled NF strains in giving a bidirectional gradient of donor marker frequencies amongst the recombinants, with obligate inheritance of a region of the donor chromosome near to 9 o'clock.

The second mode of origin of presumptive NF strains from the new donors involved 
Table 8. Non-selective analysis of a cross of $\mathrm{A} 634$, a presumptive $\mathrm{NF}$ recombinant strain (outer circle), with I098 (UF: inner circle)

Numbers represent the allele frequencies amongst the recombinants, that is excluding the arg pro cys str presumed parental class. The asterisk indicates the 9 o'clock region of the donor chromosome obligatorily included in the recombinant progeny of NF $\times$ UF crosses (Hopwood et al. I969).

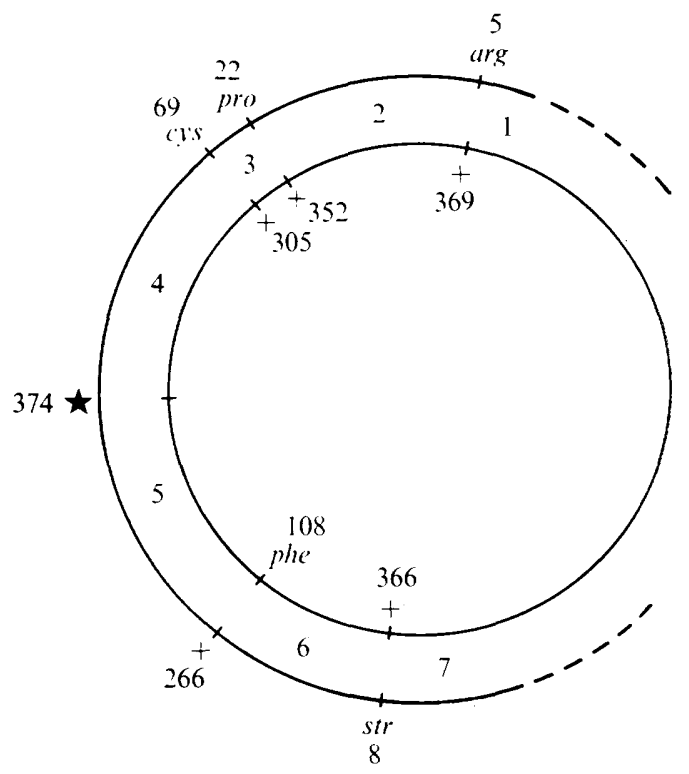

Crossovers in intervals

$\begin{array}{cc} & \text { Phenotype } \\ 5 & \text { phe } \\ 6 & - \\ 7 & \text { str }\end{array}$

Frequencies of simple crossover classes

$\begin{array}{cccc}\text { I } & 2 & 3 & 4 \\ \text { arg pro cys } & \text { pro cys } & \text { cys } & - \\ 2 & 5 & 14 & 87^{*} \\ 2 & 13 & 29 & 213 \\ (4) \dagger & 0 & 3 & 5\end{array}$

Multiple crossover classes

$\arg$ cys $\mathrm{I}$

* The fertility of these segregants, bearing the marker combination of the uf parent, was of donor type (tester strain I I 90).

$\dagger$ Presumed to be asexual.

crossing with a UF strain. As described above, the majority of the recombinants in such crosses were donors resembling the donor parent. However, a minority turned out to have the properties of NF strains in giving a bidirectional gradient of marker frequencies with a high point near the 9 o'clock region. Table 8 shows the result of crossing A634, derived from a cross of the $p a b A^{+}$donor A 620 with A372 UF against a UF strain. Nearly $99 \%$ of the progeny were non-parental, as in a typical NF $\times$ UF cross (Hopwood et al. 1969) and the pattern of marker inheritance closely resembled the NF $\times$ UF model.

Since few of the progeny of $p a b A^{+}$donor $\times$UF crosses have so far been characterized as resembling NF it is so far impossible to deduce how NF strains arise. 


\section{DISCUSSION}

The new donor strains described here resemble NF and IF strains in inhibiting aerial mycelium production by UF cultures and thus presumably still harbour the plasmid SCP I. This conclusion is confirmed by the ability of at least some of the donors to give rise to IF colonies. If, as seems likely (see Introduction), the donor properties of NF strains are due to integration of SCP I in the 9 o'clock region of the genome, then the new donors very probably derive their particular properties from a different interaction between plasmid and chromosome.

The most striking feature of the new donors is their instability. Although there was one exception (A608), instability may well be a primary characteristic of the donor strains; since the method of isolation would doubtless have favoured stable donors, the real proportion of unstable strains may well have been even higher than it seemed. Thus conceivably A608 was derived via a secondary event from an unstable donor.

In their behaviour in crosses, the new donors differ from both IF and NF strains. Unlike the IF strain from which they were derived, the new strains donate certain chromosomal markers to UF recipients with a very high frequency, nearly as high as SCP I inheritance in IF $\times$ UF crosses (Vivian, I97I) and as high as the donation of certain markers in NF $\times$ UF crosses (Hopwood et al. 1969). However, the pattern of donation differs strikingly from that characteristic of NF strains in giving a unidirectional gradient of marker frequencies from a fixed point on the linkage map; the donated fragments thus have one end fixed and the other variable. In contrast, NF strains give a bidirectional gradient, on either side of the 9 o'clock region of the genome, with both ends of the fragment of variable position (Hopwood et al. I969). Moreover the data of Hopwood et al. (I969) clearly showed that in NF $\times$ UF crosses markers on either side of 9 o'clock are frequently inherited by one and the same progeny rather than alternatively.

The two classes of new donor described here, ' $p a b A^{+}$donors' and ' $u r a A^{+}$donors', differed in the position of the fixed end of the donated fragments which was, respectively, between cys $A$ and $u r a A$, and between $u r a A$ and phe $A$. The direction of the gradient of donated marker frequencies was the same, clockwise, in both classes of donors. However, this is not characteristic of all 'unidirectional' donors, some of which have recently been derived from other IF strains, and give an anticlockwise gradient (A. Vivian \& D. A. Hopwood, unpublished results).

Virtually all the progeny of crosses of the new unstable donors with UF strains revealed the presence of SCP I by inhibiting UF aerial mycelium production. Thus, it is likely that SCP I and the point on the donor chromosome that is obligatorily inherited by the progeny, as deduced from the results of genetic analysis (Tables 4 to 7 ), are associated, just as in the case of $\mathrm{NF} \times$ UF crosses. On this hypothesis the two classes of unstable donors described here would differ in the position on the chromosome with which the plasmid is associated. However, the extreme instability of the strains, in terms of their donor ability, tends to argue against an integration of the plasmid into the chromosome, unless a very transient one. A more likely hypothesis is that these donors each harbour a still autonomous butmodified SCP I which has acquired the ability to mobilize certain chromosomal regions from the donor to a UF recipient. In Escherichia coli such strains arise by transfer of chromosomal regions to the fertility factor F, giving rise to F prime strains (Jacob \& Adelberg, 1959; Adelberg \& Burns, 1960; Hirota \& Sneath, 196I). If a corresponding mechanism is responsible for the production of the new donors of Streptomyces coelicolor, those so far detected probably do not harbour plasmids carrying the chromosomal markers whose donation has been described in this paper, since the markers are transferred with frequencies falling well below $100 \%$. Instead, 
transfer of markers could occur by a transient association between plasmid and chromosome, perhaps by 'donor crossing-over' (Campbell, I962) between a site on the chromosome corresponding to the fixed end of the population of donated fragments and a homologous region on the plasmid, or alternatively by some other kind of temporary interaction (Evanchik, Stacey \& Hayes, 1969). A reversible interaction is indicated by the finding that the progeny tend to resemble the donor parent in their fertility properties.

On this hypothesis the production of UF strains from the new donors is easily explained as due to loss of the modified autonomous plasmid, although it is surprising that this should occur so frequently, even though some long F prime strains of Escherichia coli are comparatively unstable. The spontaneous rate of loss of the normal SCP I from IF strains is about 0.03 to $0.3 \%$ (Vivian \& Hopwood, 1970 ), whereas loss of the modified plasmid from A607 and A6ro sometimes exceeded 50\% (Table 2). The ability of u.v. irradiation to increase the rate of UF production from the unstable donors, as from IF strains, is consistent with loss of a modified SCP I as an explanation of their instability. The production of IF strains, presumably carrying the normal SCP I, from the unstable donors might be explained by an internal crossover event within the modified plasmid.

The 'secondary' classes of unidirectional donors, of which the grey donor segregants from A607 are one example, while A608, although isolated in one apparent step from the original IF strain, may be a second (see above), presumably have a different explanation. Neither of these strains was observed to lose the plasmid with easily detectable frequency, nor to give rise directly to IF strains. In the case of $\mathrm{A} 607$, there was a rather frequent oscillation between the original white form that readily segregated UF and IF variants and the grey form that did not. Perhaps such 'secondary' donors arose by integration of a modified SCP I to produce a chromosomal duplication in which crossing-over had a high probability of occurrence to regenerate the autonomous plasmid. It is notable that the two forms of A607 gave virtually identical patterns of marker donation.

Also derivable from the unidirectional donors are strains resembling the type NF that arose spontaneously (Vivian \& Hopwood, 1970). The behaviour of NF strains in crosses with UF recipients (Hopwood et al. 1969) still awaits explanation. However, the discovery that strains of such type probably have a complex origin makes their more complex donor properties, as compared with the unidirectional donors described in this paper, not unexpected.

The white phenotype of most of the new donors has no obvious explanation. Their spores appeared, from cursory examination in the phase-contrast microscope, to be morphologically normal, unlike most, but not all, of the white (whi) mutants of Streptomyces coelicolor studied by Hopwood, Wildermuth \& Palmer (1970) and Chater (1972). Although UF and IF segregants from the unstable donors regained their normal grey colour, the white phenotype was separable from donor behaviour since grey donors were readily derived from A607 and could perhaps have been obtained from the other donors by further experimentation.

As we have seen, the new donors described in this paper pose a number of problems of interpretation. Distinction between plasmid borne and chromosomal genes in the way that has been done in Escherichia coli (Clowes \& Moody, 1966) is not possible in Streptomyces coelicolor since recombination deficient mutants are not yet available in this organism (Harold \& Hopwood, 1970, 1972). However, other techniques are doubtless feasible and meanwhile demonstration of the ability of SCP I to interact with the chromosome at different points and in different ways has opened the prospect of new approaches to the genetics of the organism. Some are likely to have applications in the experimental transfer of desirable properties between strains in industrial culture improvement programmes (Hopwood, 1972). 
It is a pleasure to thank Mrs Helen Tovell for excellent technical assistance. One of us (A.V.) gratefully acknowledges a postdoctoral stipend from Science Research Council grant number $\mathrm{B} / \mathrm{SR} / 4060$.

\section{REFERENCES}

Adelberg, E. A. \& Burns, S. N. (1960). Genetic variation in the sex factor of Escherichia coli. Journal of Bacteriology 79, $32 \mathrm{I}-330$.

CAMPBell, A. (1962). Episomes. Advances in Genetics II, IOI-145.

CHATER, K. F. (I972). A morphological and genetic mapping study of white colony mutants of Streptomyces coelicolor. Journal of General Microbiology 72, 9-28.

Clowes, R. C. \& Moody, E. E. M. (1966). Chromosomal transfer from 'recombination-deficient' strains of Escherichia coli $\mathrm{K} 12$. Genetics 53 , 717-726.

EvanchiK, Z., StaceY, K. A. \& HaYes, W. (1969). Ultraviolet induction of chromosome transfer by autonomous sex factors in Escherichia coli. Journal of General Microbiology 56, I-I4.

HAROLD, R. J. \& HopwoOd, D. A. (1970). Ultraviolet-sensitive mutants of Streptomyces coelicolor. I. Phenotypic characterization. Mutation Research ro, 427-438.

HAROLD, R. J. \& Hopwood, D. A. (1972). A rapid method for complementation testing of ultravioletsensitive (uvs) mutants of Streptomyces coelicolor. Mutation Research 16, 27-34.

Hirota, Y. \& SNEATH, P. H. A. (I961). $\mathrm{F}^{\prime}$ and F mediated transduction in Escherichia coli $\mathrm{K}$-I 2. Japanese Journal of Genetics 36, 307-3I8.

Hopwood, D. A. (1959). Linkage and the mechanism of recombination in Streptomyces coelicolor. Annals of the New York Academy of Sciences 81, 887-898.

Hopwood, D. A. (1967). Genetic analysis and genome structure in Streptomyces coelicolor. Bacteriological Reviews 31, 373-403.

Hopwood, D. A. (1972). Genetics of the actinomycetales. In Actinomycetales: Characteristics and Practical Importance, pp. I3I-I53. Edited by G. Sykes and F. A. Skinner. London: Academic Press.

Hopwood, D. A., Harold, R. J., Vivian, A. \& Ferguson, H. M. (1969). A new kind of fertility variant in Streptomyces coelicolor. Genetics 62, 46I-477.

Hopwood, D. A., Wildermuth, H. \& PALmer, H. M. (1970). Mutants of Streptomyces coelicolor defective in sporulation. Journal of General Microbiology 6r, 397-408.

JACOB, F. \& AdeLBERG, E. A. (1959). Transfert de caractères génétiques par incorporation au facteur sexuel d'Escherichia coli. Comptes rendues de l'Académie de sciences 249, I 89-19I.

Sermonti, G. \& Casciano, S. (1963). Sexual polarity in Streptomyces coelicolor. Journal of General Microbiology 33, 293-301.

VIVIAN, A. (1971). Genetic control of fertility in Streptomyces coelicolor A3(2): Plasmid involvement in the interconversion of UF and IF strains. Journal of General Microbiology 69, 353-364.

Vivian, A. \& Hopwood, D. A. (1970). Genetic control of fertility in Streptomyces coelicolor A3(2): the IF fertility type. Journal of General Microbiology 64, IOI-1 17. 